\title{
Tipología predial del sector productor de cerezas del sur de la Patagonia argentina
}

\author{
Righi E.; E.D. Cittadini, C. Mundet, L. San Martino, C. Sanzy N. Baltuska
}

\begin{abstract}
RESUMEN
La identificación de tipologías prediales es una herramienta útil para sintetizar la diversidad intrínseca de cada sistema de producción y puede ser eficientemente usada para realizar una selección racional y metódica de las fincas representativas en el contexto de proyectos de investigación y extensión. Sin embargo, las metodologías que se utilizan más comúnmente para producir una tipología presentan algunas características que limitan su aplicación expeditiva para la selección de fincas piloto. El objetivo de este trabajo fue realizar una identificación cuantitativa y una caracterización de tipologías prediales, sobre la base de análisis multivariado de fincas productoras de cerezas en el sur de la Patagonia argentina. Se aplicó una metodología innovadora combinando escala multidimensional, análisis de conglomerados y análisis de porcentajes de semejanza, sobre la base de la cual se identificaron seis diferentes tipos de fincas. Las ventajas de este método para la selección de fincas representativas son mostradas y discutidas a través de la individuación de una finca piloto dentro de cada tipo.
\end{abstract}

Palabras clave: caracterización cuantitativa, escala multidimensional, diversidad de sistemas de producción, análisis multivariado

Righi E.; E.D. Cittadini, C. Mundet, L. San Martino, C. Sanz and N. Baltuska, 2011. Farm typology of the sweet cherry production sector of southern Argentinean Patagonia. Agriscientia XXVIII: 85-97

\section{SUMMARY}

Farm typology identification is a useful tool to summarize the diversity that is intrinsic to every farming system and it can be efficiently used to carry out a rational and methodical selection of representative farms within the context of research and extension projects. However, the methodologies that are most commonly used to produce a typology present some features that limit its expeditious application for the selection of pilot farms. The objective of this study was to make a quantitative identification and characterization of farm typologies based on multivariate analysis of the farms of southern Argentinian Patagonia 
that grow sweet cherry. An innovative methodology which combines multidimensional scaling, cluster analysis and similarity percentages analysis was applied and six relevantly different farm types were defined. The advantages of this method for the selection of representative farms are shown and discussed by individualizing one pilot farm within each type.

Key words: quantitative characterization, multidimensional scaling, farming systems diversity, multivariate analysis

E. Righi. Dipartimento di Scienze Agronomiche e Gestione del Territorio Agroforestale dell' Università degli Studi di Firenze (DISAT-UNIFI), Piazzale delle Cascine, 18 - 50144 Firenze, Italia. E.D. Cittadini y C. Sanz. Grupo de Fruticultura, INTA-EEA Chubut, Trelew, Argentina. C. Mundet y N. Baltuska. INTA-AER Sarmiento, Sarmiento, Argentina. L. San Martino. INTA-AER LoS Antiguos, Los Antiguos, Argentina. Correspondencia a E.D. Cittadini: ecittadini@correo.inta.gov.ar

\section{INTRODUCCIÓN}

\section{Tipificación cuantitativa de predios}

Cada organización predial tiene diferentes recursos y restricciones, los cuales afectan los procesos de toma de decisiones y el manejo productivo en forma única (Kobrich et al., 2003). La identificación de tipologías prediales es una herramienta útil para lidiar con este problema, porque permite resumir la diversidad de un sistema productivo y considerarlo para el diseño de políticas o estrategias de manejo (Gaspar et al., 2008), para el desarrollo y evaluación de tecnologías innovadoras (Olaizola et al., 2008; Blazy et al., 2009a) o para la evaluación de la sustentabilidad y la calidad ambiental de los sistemas (Nahed et al., 2006; Joffre \& Bosma, 2009).

Pueden distinguirse dos métodos para identificar tipologías prediales: investigación a priori, basada en conocimiento de expertos, y tipificación cuantitativa, basada en análisis estadístico multivariado. La principal ventaja de este último reside en la objetividad, ya que se basa en la estadística, y el uso más completo de los datos disponibles (Iraizoz et al., 2007). En la mayor parte de los trabajos publicados en los que se adoptó la tipificación cuantitativa se utilizó análisis factorial, análisis de correspondencias múltiples o análisis de componentes principales para reducir el número inicial de variables a un número limitado de factores (o componentes); esto permitió lidiar con el problema de multicolinearidad entre las variables, que habitualmente dificulta la formación de conglomerados (Iraizoz et al., 2007). Sucesivamente se aplica una aglomeración jerárquica para agrupar muestras prediales (ej. Kobrich et al., 2003; Maseda et al., 2004; Milán et al., 2006; Iraizoz et al., 2007; Gaspar et al., 2008; Olaizola et al., 2008; Poussin et al., 2008; Blazy et al., 2009b).

Este enfoque mostró dos importantes limitaciones cuando hay que seleccionar predios representativos de las tipologías identificadas. La metodología basada en análisis de conglomerados (AC) falla en la representación de las interrelaciones entre las muestras en una escala continua, cuando muestras y agrupamientos que son representados cerca uno de otro en un dendograma AC pueden ser en realidad muy diferentes. El AC agrupa las muestras dentro de conglomerados discretos, pero no es capaz de mostrar una graduación estable en la estructura de la base de datos y tiende a imponer agrupamientos bastante arbitrarios en lo que parecen ser cambios continuos (Clarke \& Warwick, 2001). Además, el uso de factores o componentes para definir los conglomerados enmascara la comprensión sobre cuáles son las principales variables responsables de la clasificación. En este sentido, faltan estudios sobre identificación de tipologías, incluso si los problemas pueden ser abordados estudiando la correlación de las variables con los componentes principales (e.g. Gaspar et al., 2008). Estos hechos limitan fuertemente su utilización expeditiva para la identificación de fincas representativas.

Las limitaciones descritas previamente podrían ser resueltas mediante la combinación de AC, escala multidimensional (EMD) y análisis de porcentajes de semejanzas (similarity percentages: SIM- 
$P E R)$. Mapas de ordenación EMD revelan un continuo natural dentro de los grupos definidos por el AC y así limitan el riesgo de subdivisiones arbitrarias de las muestras. También tienen mayor capacidad para representar con precisión relaciones más complejas en espacios bidimensionales (Clarke \& Warwick, 2001). El análisis SIMPER es una técnica de análisis multivariado usada para estudiar la contribución de las variables a la semejanza dentro de los conglomerados, que provee información sobre las variables más representativas para definir una tipología. En este artículo se argumenta que este enfoque es particularmente apropiado para generar una tipología predial con el objetivo de seleccionar fincas piloto representativas.

El cultivo de cerezos (Prunus avium L.) ha sido identificado como una de las actividades agropecuarias más promisorias para el sur de la Patagonia argentina (específicamente para las provincias de Chubut y Santa Cruz) en función de su favorable comportamiento productivo en la región y las potencialidades comerciales identificadas (producción en contra estación con respecto al hemisferio norte). Al mismo tiempo, la alta demanda de mano de obra es considerada por los funcionarios políticos como una característica positiva.

El cultivo tuvo un importante desarrollo en la última década, impulsado por políticas de promoción para el sector. Sin embargo, en las últimas temporadas la rentabilidad ha disminuido como producto de los bajos rendimientos y calidad de fruta y del deterioro de los precios relativos del producto con respecto a los insumos.

EI Proyecto EULACIAS (EUropean Latin American Co-Innovation for Agricultural Systems) se desarrolló entre febrero de 2007 y julio de 2010. Se basó en tres dominios de conocimiento que interactúan entre sí: sistemas complejos, aprendizaje social y monitoreo dinámico, y evaluación de proyectos. El proyecto se planteó como meta la identificación y el desarrollo, junto con productores, funcionarios y técnicos, de sistemas alternativos de producción de frutas y de opciones de política que permitan el incremento del ingreso predial y el uso sustentable de los recursos naturales, utilizando un enfoque ecosistémico y metodologías participativas.

El estudio de caso argentino (el proyecto incluye otros dos casos: horticultura periurbana en Montevideo, Uruguay, y lechería minifundista en Michoacán, México) concentró los esfuerzos en fincas piloto en las tres principales áreas de producción del sur de la Patagonia: Valle Inferior del Río Chubut (VIRCh), Sarmiento y Los Antiguos, con la intención de aplicar luego los resultados en un universo más amplio de fincas de la región.

Una selección arbitraria de predios para participar de este proyecto, sin una adecuada caracterización y segregación, hubiese limitado la validez de los resultados obtenidos y hubiese restringido la efectividad de las medidas de intervención diseñadas. Por lo tanto, se hizo necesario un enfoque integral para identificar grupos de predios (tipos) con características diferenciadas. El agrupamiento de los predios en 'tipos' permitió hacer extensivas las recomendaciones que surgieron de las investigaciones en el marco del proyecto EULACIAS a otros predios no participantes, pero que presentaban características que permitían incluirlos en algunos de los grupos identificados.

\section{La producción de cerezas en el sur de la Pata- gonia argentina}

En las provincias de Santa Cruz y Chubut el área total con cerezos se ha incrementado de 176 ha en 1997 a 580 ha a principios de 2009, de las cuales 200 están en Los Antiguos (46 19' S; 220 msnm), 175 ha en el Valle Inferior del Río Chubut (VIRCh) (43 16' S; 30 msnm), 142 ha en Sarmiento

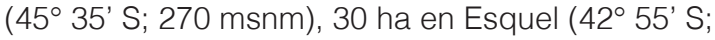
570 msnm) y 33 ha en Comodoro Rivadavia (45 52 ' S; 50 msnm). Las variedades más comunes son 'Lapins', 'Bing', 'Sweetheart', 'Newstar', 'SteIla', 'Sunburst' y 'Van' injertadas sobre 'Mahaleb', 'SL64', 'Pontaleb', 'Colt' y 'Mazzard' (Cittadini et al., 2008). Los árboles generalmente son regados por goteo y plantados en alta densidad, con sistemas de conducción como tatura, eje central y vaso modificado (2700, 1100 y 1000 árboles ha-1 $^{-1}$, respectivamente). Sin embargo, también están presentes otros sistemas de riego menos eficientes (gravitacionales) y sistemas de conducción más tradicionales, con densidades de plantación media y baja. Hay diferentes sistemas de control de heladas, de los cuales el riego por aspersión es el más efectivo. La adopción de estos sistemas varía de acuerdo con las características climáticas de cada zona y las percepciones con respecto al riesgo por parte de los productores. Las cerezas son cosechadas desde noviembre (VIRCh) hasta fines de enero (Los Antiguos y Esquel), y sólo para cosecha la demanda de mano de obra durante la temporada 2006/2007 fue de 190.000 horas. En esa temporada, 11 empaques exportaron 729 toneladas (45\% del total producido) a Europa. La mayoría de los montes aún no han alcanzado su techo de producción y algunos nuevos se están implantando. Por lo tanto, los volúmenes de fruta continuarán incrementándose, por lo que la disponibilidad de mano de obra y la capacidad de empaque pueden con- 
vertirse en restricciones para el desarrollo regional.

El objetivo de esta investigación fue categorizar fincas orientadas a la producción de cerezas como actividad principal, para definir tipologías y evaluar la utilidad de esto para la selección de fincas piloto representativas de los principales grupos en programas de investigación y transferencia, en un contexto de limitada disponibilidad de recursos para la intervención.

\section{MATERIALES Y MÉTODOS}

\section{Muestreo y recolección de datos}

El muestreo de fincas para el presente análisis representa $83,2 \%$ del área total con cerezos en la región: 30 fincas en el VIRCh, 34 en Los Antiguos y 17 en Sarmiento. La mayoría de los productores de estas fincas ya tenían alguna interacción con los servicios de extensión y los programas de investigación del INTA, o con servicios privados de asesoramiento, por lo que había disponible una gran cantidad de datos a nivel predial relativamente recientes, los cuales estaban sistematizados en bases de datos, informes técnicos o publicaciones (Muñoz, 2004; Sanz, 2005; Cittadini et al., 2008). Los datos sobre mano de obra temporaria estaban incompletos, por lo cual fueron estimados mediante el programa FRUPAT (Cittadini, 2005), que es una herramienta para cuantificación de insumos y productos de los sistemas de producción de frutas del sur de la Patagonia (Cittadini et al., 2006).

\section{Protocolo de análisis: procedimiento para la identificación de tipologías prediales}

El procedimiento que se utilizó en este estudio se desarrolló dentro del contexto de proyectos de investigación y desarrollo que tomaron en cuenta un gran número de estudios de caso en diferentes países (Pacini et al., 2006; Righi et al., 2009). En el contexto de estos proyectos, se estableció a priori un marco común para la selección de variables de clasificación, con el propósito de permitir la comparación de resultados entre los diferentes estudios de caso. En este sentido, se consideraron tres categorías generales de variables (CGV) como esenciales para construir las tipologías teniendo en cuenta la diversidad en el análisis sistémico cuantitativo y en el diseño de estrategias innovadoras a nivel de finca. Las CGV se definieron como: (i) recursos biofísicos, (ii) aspectos socioeconómicos y (iii) equipamiento. La primera categoría incluyó variables como área total de la finca, patrones de uso de la tierra, recursos ganaderos, plantaciones de frutales y escala del sistema de producción. La segunda comprendió variables de trabajo, capital, manejo, organización y aspectos sociales de la persona responsable del sistema. La tercera categoría se relacionó con la dotación de tecnología, infraestructura y disponibilidad de herramientas y medios para el proceso productivo. El objetivo de las CGV fue crear un marco para la construcción de una base de datos que fuera aplicable a diferentes sistemas de producción y que permitiera la comparación de los resultados (Pacini et al., 2006; Righi et al., 2009; Righi et al., 2010). A partir de ello, expertos locales definieron un set de variables de clasificación específico para cada estudio de caso, cubriendo los tópicos resaltados por las CGV, teniendo en cuenta la estructura del sistema productivo e identificando las fuentes de variación más importantes entre las fincas.

Para caracterizar la estructura predial y el funcionamiento de los distintos sistemas de producción de cerezas del sur de la Patagonia, se utilizó información disponible en informes técnicos y artículos científicos y se realizaron consultas complementarias con los productores. Posteriormente, se confeccionó una lista preliminar de variables de clasificación, las cuales se enumeran a continuación:

Tierra y montes

- Área total de la finca (ha)

- Área total con cerezos (ha).

- Densidad de plantación de cerezos (árboles/ ha).

\section{Aspectos socioeconómicos}

- Año inicial de plantación de los montes de cerezo (año).

- Mano de obra permanente (cantidad de empleados).

- Mano de obra transitoria (jornales: $8 \mathrm{~h}$ ).

- Asesoramiento (no: 0; ocasional: 1; permanente: 2).

- Certificación de procesos o productos (no: 0; implementación de protocolos: 1; certificación de protocolos: 2).

- Empaque (no: 0; en forma asociada: 1; propio: 2).

\section{Equipamiento y tecnología}

- Sistema de riego (porcentaje del área con riego por goteo con respecto al área total irrigada).

- Control de heladas mediante riego por aspersión (porcentaje del área protegida con respecto al área total implantada con cerezos).

- Número de tractores.

- Número de pulverizadoras. 
La lista de variables clasificatorias incluyó tanto variables cuantitativas como cualitativas. Las cualitativas se trataron como variables discretas cuantitativas atribuyéndoles rangos a las diferentes clases. Se asumió una tendencia cualitativa específica entre clases tal que, por ejemplo, la clase 2 era mejor que la 1 y que la clase 1 era mejor que la 0 . La proporcionalidad de los valores entre las diferentes clases de las variables cualitativas fue definida por expertos. La identificación de las tipologías se basó en un pequeño número de variables clave, tal lo sugerido por Kostrowicki (1977), las que fueron seleccionadas de un set inicial de variables clasificatorias. La decisión de qué variables dejar y cuáles eliminar fue llevada a cabo por expertos y sustentada en el análisis de componentes principales (ACP) exploratorio. Se aplicó un ACP no normado. Los datos de las variables iniciales fueron estandarizados dividiendo los valores absolutos por el total de cada variable para neutralizar el efecto de la unidad de medida.

Para calcular la matriz de semejanzas se utilizó el método de Bray-Curtis (Bray \& Curtis, 1957). La matriz de semejanzas contiene los coeficientes de semejanza calculados entre cada par de individuos dentro de una muestra. Convencionalmente, un coeficiente de semejanza toma valores en un rango de $0-100 \%$. El coeficiente es igual a $100 \%$ si dos individuos son totalmente similares, y es igual a 0 si son totalmente disímiles (Clarke \& Warwick, 2001). El método de Bray-Curtis es un coeficiente no métrico que puede ser aplicado con variables multidimensionales, estandarizadas, cuantitativas y cualitativas. Los coeficientes de semejanza de la matriz son los datos de base para las técnicas de análisis multivariado, escala multidimensional $(E M D)$ y análisis de conglomerados (AC). Este procedimiento ha sido adaptado de un método desarrollado en el Plymouth Maine Laboratory para el análisis de cambios en comunidades marinas (Clarke \& Warwick, 2001). Una metodología similar ha sido aplicada para clasificar sitios en ecología y biología (ej. Catalán et al., 2006; Stergiou et al., 2006; Selleslagh \& Amara, 2008). Como ha sido mostrado en otros estudios de caso en diferentes regiones de África y América Latina (Pacini et al., 2006; Righi et al., 2009, Righi et al., 2010), sus aplicaciones mostraron ser lo suficientemente robustas como para ser también utilizadas para bases de datos de muestras prediales.

Las tipologías prediales fueron generadas usando una combinación de los resultados de EMD y AC. El análisis de semejanzas usando EMD es un método de ordenamiento cuyo propósito es construir un "mapa" o configuración de las muestras, en el que los resultados se presentan en gráficos de dos dimensiones (Kruskal, 1964; Kruskal \& Wish, 1978). La interpretación se realiza en términos de las distancias relativas de una muestra a otra, debido a que los rangos de semejanza son la única información utilizada por la ordenación EMD no métrica. Para un conjunto de datos realista normalmente hay alguna distorsión en la ordenación que es minimizada mediante el algoritmo EMD. Su medida se denomina valor de estrés (Clarke \& Warwick, 2001).

Se utilizó un conglomerado jerárquico para agrupar fincas según el método de enlace de grupo promedio (Everitt, 1980; Field et al., 1982). El análisis de semejanzas mediante $\mathrm{AC}$ es un método multivariado que tiene por objeto encontrar "agrupamientos naturales", de modo que muestras dentro de un conglomerado sean más parecidas entre sí que muestras en conglomerados diferentes. Los resultados de este análisis son presentados en dendogramas (Field et al., 1982; Clarke \& Warwick, 2001).

El número de conglomerados fue determinado mediante un procedimiento heurístico (Kobrich et al., 2003; Righi et al., 2010), a través de una evaluación subjetiva del dendograma AC. El número de conglomerados se definió por un nivel mínimo de semejanza dentro de los grupos. Los grupos se visualizaron en el gráfico de EMD dibujando círculos alrededor de las fincas de un mismo conglomerado.El último paso metodológico fue el análisis SIMPER de los conglomerados tipológicos finales (Clarke, 1993). El análisis SIMPER se concentra en las semejanzas Bray-Curtis entre muestras y resalta las principales variables responsables de la determinación de los agrupamientos de muestras en un análisis de conglomerados. El algoritmo SIMPER primero computa la semejanza media entre todos los pares de muestras dentro de un grupo y entonces desagrega dicho promedio en contribuciones separadas de cada variable, de acuerdo a las semejanzas medias de la variable, calculadas con el coeficiente de Bray-Curtis. Tal coeficiente es concebido de modo que las variables cuyos valores son todos iguales a cero dentro de un grupo (por ejemplo "Área con riego por goteo", en un grupo en el que ninguna finca utiliza dicho sistema de riego), no contribuyen a la semejanza media intragrupal de acuerdo al análisis SIMPER.

Los valores medios de las variables cuantitativas de los agrupamientos prediales más representativos fueron usados para construir una tipología. Para las variables cualitativas se utilizó la moda para representar a un grupo. La dotación de recursos de cada tipo de finca se resumió en una finca virtual 
de grupo (FVG) compuesta por las características medias y modales de todo el grupo. Las variables que proveyeron las contribuciones más altas para formar los conglomerados de acuerdo al análisis SIMPER fueron puestas en evidencia para caracterizar los agrupamientos como tipos formales.

Todos los análisis estadísticos requeridos en esta investigación fueron realizados con el programa PRIMER 6.

\section{Selección de fincas representativas}

Una finca representativa, dentro de cada tipo de finca, se identificó por medio del análisis SIMPER, EMD y AC. Como el análisis SIMPER provee información de las variables más importantes que caracterizan a un conglomerado, la selección de fincas representativas mediante SIMPER incluyó a todas aquellas fincas que tenían valores muy similares a los de la FVG en las variables que presentaron la mayor contribución a la semejanza dentro de grupo.

Se realizaron EMD y AC adicionales incluyendo a la FVG en la base de datos para identificar a las fincas candidato representativas. Todas las fincas que representaron adecuadamente a las características promedio de un tipo de finca mostraron niveles muy altos de semejanza con la correspondiente FVG en las representaciones gráficas de EMD y AC (Righi et al., 2010).

\section{RESULTADOS Y DISCUSIÓN}

\section{Selección de variables clave}

Las 13 variables de clasificación presentadas en la sección anterior se sometieron a un ACP para sustentar la elección de variables clave para la identificación de tipos. Los resultados del ACP se muestran en la Tabla 1. Los primeros tres CP explicaron el $38,9 \%, 26,2 \%$ y $10,5 \%$ de la varianza total, mientras que los CP 1-5 en conjunto explicaron el $88,4 \%$. La variable 'área total' tuvo la mayor contribución a los ejes CP1 y CP2, revelando la contribución más importante en las representaciones del ACP. Sin embargo, esta variable fue eliminada de los análisis posteriores ya que la mayoría de las fincas tenían grandes áreas y las plantaciones de cerezos sólo ocupaban una pequeña porción del total de la superficie de la finca. En este sentido, la disponibilidad de tierra no es en general una restricción para la región. Como la variable habría tenido un fuerte impacto en la discriminación de finca, no se incluyó entre las variables clave.

La variable 'año inicial de plantación' se descartó debido a que no tuvo influencia en la descripción de la varianza muestral, y tuvo niveles de correlación muy bajos con los cinco primeros CP (Tabla 1). Las variables 'asesoramiento' y 'certificación de procesos o productos' se correlacionaron entre sí. Por este motivo se decidió mantener sólo una de ellas, y la elección se basó en el conocimiento de

Tabla 1. Resultados del análisis de componentes principales realizado con las 13 variables de clasificación consideradas para la identificación de tipologías prediales del sector productor de cerezas del sur de la Patagonia argentina.

\begin{tabular}{|c|c|c|c|c|c|}
\hline Componentes principales (CPs) & PC1 & PC2 & PC3 & PC4 & PC5 \\
\hline Vectores propios & 14 & 9,45 & 3,79 & 2,54 & 2,01 \\
\hline Porcentaje de la varianza explicada & 38,9 & 26,2 & 10,5 & 7,1 & 5,6 \\
\hline Porcentaje de la varianza acumulada & 38,9 & 65,2 & 75,7 & 82,8 & 88,4 \\
\hline \multicolumn{6}{|l|}{ Variables } \\
\hline Área total de la finca (ha) & 0,948 & $-0,242$ & 0,172 & $-0,069$ & $-0,048$ \\
\hline Área total con cerezos (ha) & 0,107 & 0,384 & $-0,209$ & $-0,343$ & 0,098 \\
\hline Año inicial de plantación de los montes de cerezo (año) & 0 & 0 & $-0,001$ & 0,001 & 0 \\
\hline \multirow{2}{*}{$\begin{array}{l}\text { Densidad de plantación de cerezos (árboles/ha) } \\
\text { Sistema de riego (\% del área con riego por goteo con respecto al área } \\
\text { total irrigada) }\end{array}$} & 0,038 & 0,024 & $-0,017$ & 0,189 & 0,084 \\
\hline & 0,057 & 0,077 & 0,013 & 0,314 & 0,056 \\
\hline $\begin{array}{l}\text { Control de heladas mediante riego por aspersión (porcentaje del área } \\
\text { protegida con respecto al área total implantada con cerezos) }\end{array}$ & 0,031 & 0,376 & 0,342 & 0,445 & $-0,591$ \\
\hline Cantidad de tractores & 0,06 & 0,171 & 0,184 & $-0,168$ & 0,183 \\
\hline Cantidad de máquinas pulverizadoras & 0,018 & 0,28 & 0,35 & 0,007 & 0,655 \\
\hline Mano de obra permanente (cantidad de empleados) & 0,265 & 0,587 & $-0,609$ & 0,029 & $-0,054$ \\
\hline Mano de obra transitória (jornales: 8 h) & 0,029 & 0,388 & 0,401 & 0,056 & 0,084 \\
\hline \multirow{3}{*}{$\begin{array}{l}\text { Asesoramiento (no: 0; ocasional: 1; permanente: 2) } \\
\text { Certificación de procesos o productos (no: 0; implementación: 1; } \\
\text { certificación: 2) } \\
\text { Empaque (no: 0; en forma asociada: 1; propio: 2) }\end{array}$} & $-0,039$ & 0,054 & $-0,046$ & $-0,113$ & $-0,093$ \\
\hline & $-0,085$ & 0,085 & 0,222 & $-0,69$ & $-0,378$ \\
\hline & $-0,008$ & 0,182 & 0,265 & $-0,155$ & $-0,07$ \\
\hline
\end{tabular}


expertos, quienes consideraron que uno de los aspectos del asesoramiento era la implementación de procesos de certificación, por lo cual 'asesoramiento' es una variable más amplia.

De las variables iniciales de 'equipamiento y tecnología', las variables 'riego' y 'control de heladas' fueron preferidas a 'número de tractores' y 'número de pulverizadoras'. Las dos primeras se seleccionaron debido a que son tecnologías que incrementan y estabilizan significativamente la producción y los ingresos, y que al mismo tiempo representan una inversión significativa. En cambio, 'número de tractores' y 'número de pulverizadoras' tienen baja variabilidad y su magnitud no tiene tanto impacto en el resultado productivo y económico de la finca.

Finalmente, se utilizaron ocho variables clave para la identificación de los tipos:

1. Área total con cerezos.

2. Densidad de plantación de cerezos (fuertemente relacionado con el sistema de conducción).

3. Mano de obra permanente.

4. Mano de obra transitoria.

5. Asesoramiento.
6. Empaque.

7. Sistema de riego.

8. Sistema de control de heladas.

\section{Tipologías prediales}

El valor de estrés (resultados de EMD -Figura 1-) fue 0,16. De acuerdo a la regla empírica para ordenamientos bidimensionales, dada por Clarke \& Warwick (2001), cuando el valor de estrés está dentro del rango 0,1-0,2 significa que el gráfico bidimensional es potencialmente útil. En el gráfico EMD (Figura 1) las fincas semejantes fueron agrupadas dentro de seis círculos de acuerdo a los resultados de AC. Los conglomerados fueron inicialmente definidos en el dendograma AC a un nivel de semejanza de 68\% (Figura 2) y representados en un gráfico EMD. El nivel de semejanza de $68 \%$ es el mínimo coeficiente de semejanza que pudo encontrarse entre fincas del mismo conglomerado.

Los tipos de fincas fueron descritos mediante la abundancia promedio y los valores modales de las variables clave dentro de cada conglomerado (Tabla 2). En el conglomerado 1 se incluyeron principalmente fincas del VIRCh, con superficies

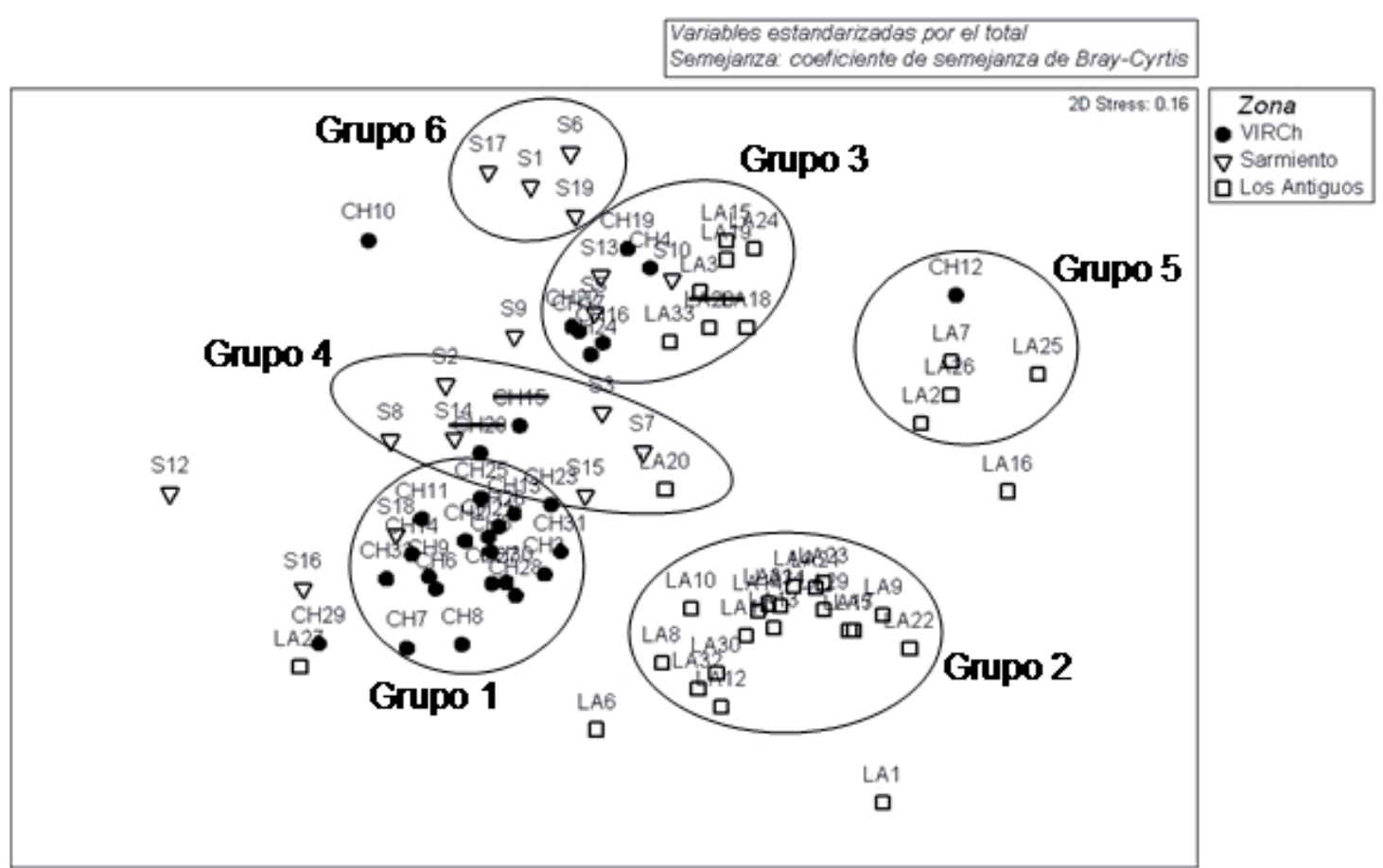

Fig. 1: Tipología de fincas orientadas a la producción de cerezas en el sur de la Patagonia. Los agrupamientos representados en el gráfico fueron identificados mediante el solapamiento de los resultados de los análisis EMD y AC, a un nivel de semejanza del 68\%. Algunas muestras no fueron incluidas en ningún conglomerado o fueron eliminadas (tachadas) debido a que formaban conglomerados de una sola finca o porque se ubicaban en la intersección entre dos conglomerados y su ubicación inequívoca no era posible. Los símbolos representan la zona de producción de cada finca: (1) círculos sólidos = VIRCh; (2) triángulos = Sarmiento; (3) cuadrados = Los Antiguos. 
Tabla 2. Valores de abundancia promedio de variables clave calculados para el agrupamiento de las fincas orientadas a la producción de cerezas en el sur de la Patagonia Argentina

\begin{tabular}{|c|c|c|c|c|c|c|}
\hline Indicador & & & Conglom & nerado & & \\
\hline & 1 & 2 & 3 & 4 & 5 & 6 \\
\hline Área total con cerezos (ha) & 4,5 & 5,50 & 2,22 & 3,69 & 1,16 & 2,12 \\
\hline Densidad de plantación de cerezos (árboles/ha) & $1795^{a}$ & 767 & 2003 & 1803 & 902 & 2406 \\
\hline Mano de obra permanente (cantidad de empleados) & 1,6 & 0,22 & 0,87 & 2,29 & 0,00 & 0,25 \\
\hline Mano de obra transitoria (jornales) & $480^{a}$ & 122 & 66 & 333 & 32 & 101 \\
\hline Sistema de control de heladas (\% con aspersión) & $88,8^{a}$ & 2,2 & 0,0 & 0,0 & 0,0 & 0,0 \\
\hline Sistema de riego (\% con de goteo) & $100^{\text {a }}$ & 0,06 & 100 & 100 & 0 & 100 \\
\hline Asesoramiento (no: 0; ncasional: 1; permanente: 2) ${ }^{b}$ & $2(54,5)$ & $2(61,1)$ & $2(53,3)$ & $1(57,1)$ & $1(80,0)$ & $0(100)$ \\
\hline Empaque (no: 0; en forma asociada: 1; propio: 2) ${ }^{\text {b }}$ & $1^{\text {a }}(72,7)$ & $1(88,9)$ & $0(100)$ & $1(71,4)$ & $0(100)$ & $0(100)$ \\
\hline
\end{tabular}

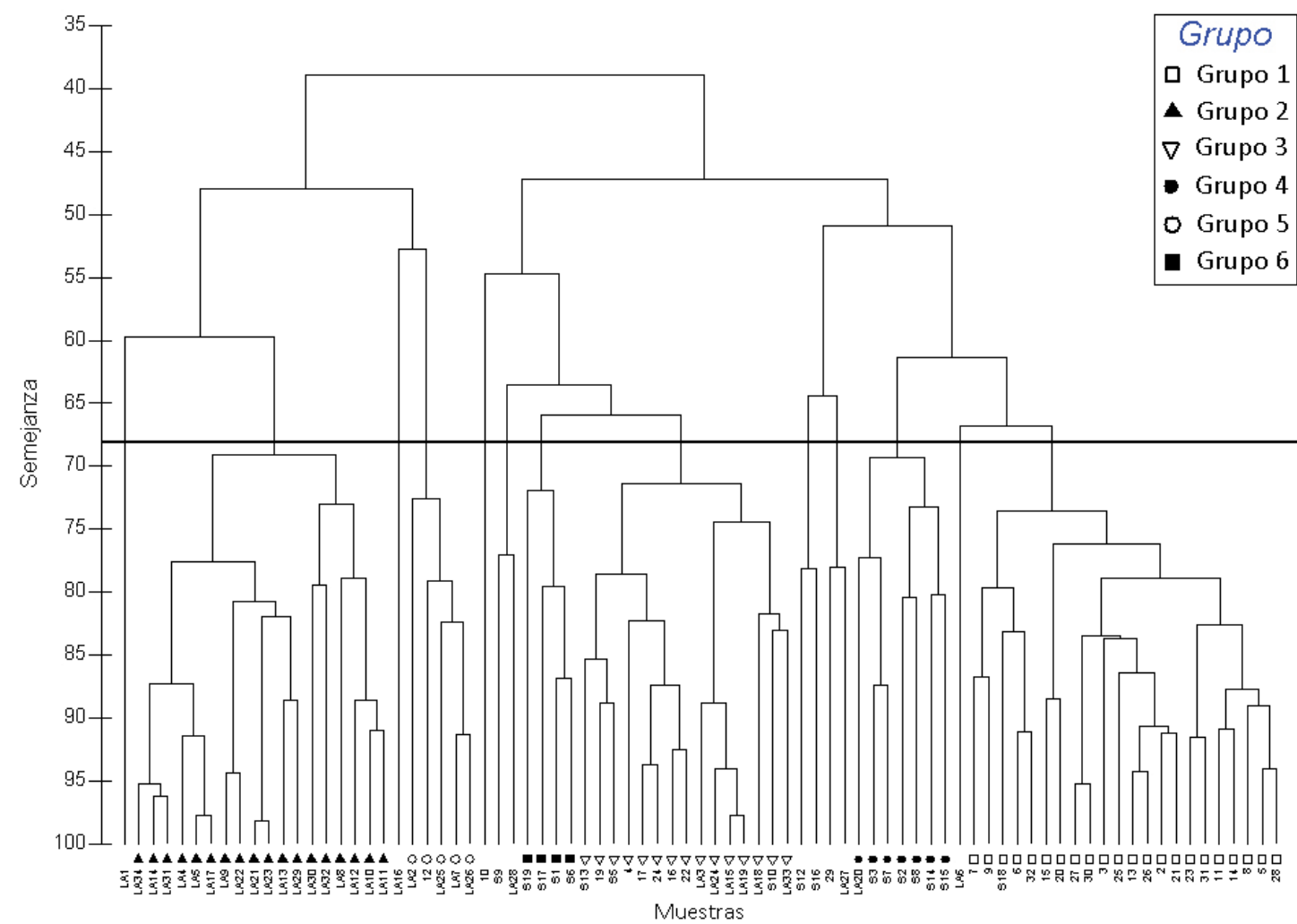

Fig. 2: Dendograma de análisis de conglomerados usado para definir los grupos prediales del sector productor de cerezas del sur de la Patagonia argentina. La línea horizontal representa el nivel de semejanza (68\%) al cual el dendograma fue "cortado" para definir los conglomerados.

implantadas relativamente importantes, alta densidad de plantación, importante uso de mano de obra, alto nivel de organización en términos de asesoramiento y empaque y buen nivel tecnológico (sistemas de riego y de control de heladas).

El conglomerado 2 estuvo constituido completamente por fincas de Los Antiguos, con el área total media con cerezos más alta, pero con la menor densidad de plantación. La demanda de mano de obra se restringía básicamente a empleados temporales. La gran mayoría de las fincas de este conglomerado tenían algún tipo de asesoramiento (61\% tenían asesoramiento permanente) y $88,9 \%$ de las fincas compartían empaques. Por el contra- 
rio, se caracterizaban por un bajo nivel tecnológico evidenciado en la casi total ausencia de equipos de riego por goteo y de control de heladas.

El conglomerado 3 incluyó fincas de las tres zonas de producción, en las cuales los sistemas de control de heladas y la organización para el empaque estaban completamente ausentes. Esto se explica por el hecho de que todas estas fincas eran plantaciones recientes que aún no estaban en producción. El área promedio era de 2,22 ha, con alta densidad de plantación. El uso de mano de obra permanente era relativamente común, pero la demanda total era baja debido a que aún no había cosecha. Todas estas fincas estaban regadas por goteo y en general contaban con asesoramiento profesional.

El conglomerado 4 incluyó principalmente a fincas de Sarmiento, caracterizadas por una superficie relativamente grande con cerezos, alta densidad de plantación y un uso importante de mano de obra. Todos utilizaban sistema de riego por goteo, pero ninguno contaba con sistema de control de heladas. La mayoría de las fincas de este conglomerado (71,4\%) manejaban el empaque en forma asociativa, mientras que otras $(28,6 \%)$ tenían su propia planta de empaque. Por el contrario, el asesoramiento era poco común.

El conglomerado 5 incluyó cinco fincas, cuatro de las cuales están en Los Antiguos. En este conglomerado los montes eran muy pequeños, plantados en baja densidad y poco demandantes de mano de obra. Ninguna de estas fincas contaba con sistema de control de heladas ni de riego por goteo. Tenían asesoramiento, pero no contaban con acceso organizado a empaques.

El conglomerado 6 fue el más pequeño, incluyendo a cuatro fincas de Sarmiento, con montes intermedios en área implantada con cerezos (2,12 ha), la más alta densidad de plantación y con riego por goteo en todos los casos. Sin embargo, la demanda de mano de obra era baja y no contaban con asesoramiento, sistema de control de heladas ni empaque.

Algunas fincas demostraron características particulares en comparación con la población. Cada una de las fincas Ch10, LA1, LA6 y LA16 representaba un conglomerado; los conglomerados prediales Ch29-LA27, S12-S16 y S9-LA28 contenían sólo dos fincas cada uno. Las características de estas fincas se muestran en la Tabla 3. Se presentan los promedios para los conglomerados que incluyeron dos fincas.

La finca Ch10 fue similar al Grupo 6, pero tenían una mayor área con cerezos y, en consecuencia, un mayor número de empleados permanentes. Las fincas LA1, LA6 y LA16 tenían muy baja densidad de plantación y bajo suministro de mano de obra, sugiriendo un sistema de producción más extensivo y familiar, lo cual podría implicar una estrategia predial diferente al resto.

Los tres conglomerados formados por dos fincas se caracterizaron por una elevada superficie con cerezos, alta densidad de plantación y alto nivel de dotación de equipamiento. El conglomerado constituido por las fincas S9-LA28 se representó bastante cerca del Grupo 3 en el gráfico de EMD, con la mayor diferencia en una mayor superficie con cerezos.

Se observó una tendencia a que las fincas ubicadas en una zona de producción de cerezas determinada pertenecieran al mismo conglomerado. Esto significa que el proceso de tipificación fue capaz de determinar cierta estructura subyacente de los sistemas productivos y que se desarrolló una tipología válida (Kobrich et al., 2003). De hecho, los productores de Los Antiguos son más tradicionales, plantando principalmente en vaso (400 a 1000 árboles ha-1) o como sistema libre (280 árboles ha-1) y regando por gravedad (74 \% del área). Sólo el 3,3 \% del área de Los Antiguos está protegida contra heladas, ya que los productores confían fuertemente en el efecto moderador del lago Buenos Aires (2240 $\mathrm{km}^{2}$, incluyendo el área en territorio chileno). En

Tabla 3. Valores de las variables claves de las fincas que demostraron características particulares en comparación con la población del sector productor de cerezas del sur de la Patagonia Argentina

\begin{tabular}{lccccccc}
\hline Variables / códigos de las fincas descartadas & Ch10 & LA1 & LA6 & LA16 & S9 - LA28 & S12 & - S16 Ch29 - LA27 \\
Área total con cerezos (ha) & 6 & 2 & 2,5 & 10 & 10,5 & 28,5 & 29,1 \\
Densidad de plantación de cerezos (árboles/ha) & 2970 & 500 & 600 & 414 & 1423,36 & 1867,16 & 1191,4 \\
Sistema de riego (\% con de goteo) & 100 & 0 & 0 & 0 & 92,5 & 100 & 100 \\
Sistema de control de heladas (\% con aspersión) & 0 & 0 & 100 & 0 & 0 & 100 & 75 \\
Mano de obra permanente (cantidad de empleados) & 6 & 0 & 0 & 0 & 2 & 17 & 6,5 \\
Mano de obra transitoria (jornales) & 0 & 0 & 80 & 0 & 110,5 & 212,5 & 1793 \\
Asesoramiento (no: 0; ocasional: 1; permanente: 2) & 0 & 0 & 2 & 1 & $1 / 1$ * & $2 / 2^{*}$ & $1 / 2^{*}$ \\
Empaque (no: 0; en forma asociada: 1; propio: 2) & 0 & 1 & 1 & 0 & $0 / 0^{*}$ & $0 / 2^{*}$ & $1 / 2^{*}$ \\
\hline
\end{tabular}

* Para las variables cualitativas 'asesoramiento' y 'empaque' se informaron dos valores por cada finca. 
Comodoro Rivadavia no se han instalado sistemas de control de heladas, debido a que los montes que ya están en producción están localizados cerca del mar, en un área con bajo riesgo de heladas. El área protegida contra heladas es del $71,4 \%$ en Sarmiento, $35 \%$ en Esquel y 83,5 \% en el VIRCh.

\section{Selección de fincas representativas}

Los valores medios de los conglomerados de fincas (Tabla 2) que fueron usados para caracterizar las tipologías prediales definen una finca virtual de grupo (FVG) por tipo de finca. En el gráfico EMD (Figura 3) la FVG de cada tipología es representada junto con todas las muestras prediales incluidas en el análisis. Debido a que la interpretación del mapa de configuración EMD se basa en las distancias relativas entre muestras, se puede asumir que las muestras que están más cerca de la FVG son las más apropiadas para representar una tipología predial. Sin embargo, para fortalecer los resultados de la EMD, las muestras prediales más similares fueron individualizadas mediante un AC. Los valores de las variables clave fueron comparados con los valores medios de la tipología correspondiente
(Tabla 4). Los resultados del análisis SIMPER fueron usados para comparar características de fincas piloto con la correspondiente finca tipo. En la Tabla 4, para cada tipo de finca, se presentaron las variables en las cuales la contribución acumulada a la semejanza dentro del grupo fue de al menos $80 \%$.

Para cada tipo se individualizó una muestra predial representativa, con valores similares a los promedios del grupo. La finca Ch21 fue seleccionada como representativa de la tipología predial 1. Dicha finca fue muy similar a la FVG para todas las variables, excepto para la densidad de plantación de cerezos, que tuvo un valor más alto que el promedio grupal.

La finca muestral LA14 fue muy buena representante del grupo 2 , sin ninguna diferencia relevante con respecto a los promedios grupales. La selección de fincas representativas para las tipologías 3 y 4 fue más dificultosa. De hecho, en el gráfico EMD, la FVG de estos dos grupos está bastante separada de las muestras prediales y más de una de ellas está a una distancia similar. Sin embargo, el AC individualizó la muestra predial más parecida al promedio grupal (finca LA33 y

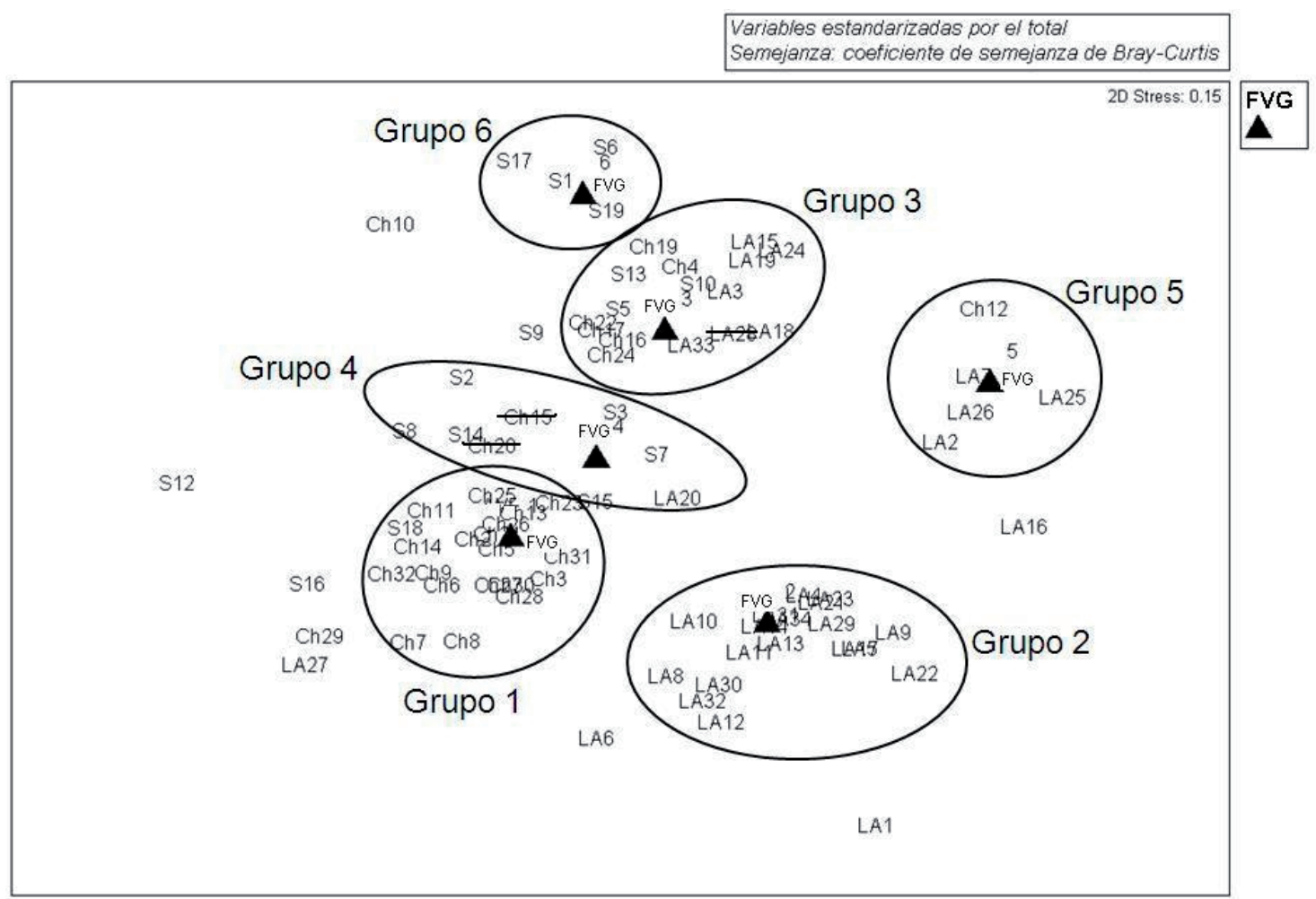

Fig. 3: Representación en escala multidimensional de las seis tipologías prediales identificadas para el sector productor de cerezas del sur de la Patagonia argentina y las correspondientes fincas virtuales de grupo (FVG; triángulos) definidas por los valores medios de cada conglomerado. 
Tabla 4. Resultados del análisis SIMPER de las tipologías prediales en comparación con las características del predio representativo seleccionado para cada una de ellas por el sector productor de cerezas del sur de la Patagonia Argentina.

\begin{tabular}{|c|c|c|c|c|c|}
\hline Indicadores ${ }^{a}$ & $\begin{array}{l}\text { Predio } \\
\text { piloto }^{b}\end{array}$ & $\begin{array}{l}\text { Promedio } \\
\text { grupalc }^{\mathrm{c}}\end{array}$ & $\mathrm{SM}^{\mathrm{d}}$ & $\begin{array}{l}\text { Contribución a la } \\
\text { semejanza dentro del } \\
\text { conglomerado (\%) }\end{array}$ & $\begin{array}{c}\text { Contribución } \\
\text { acumulada }(\%)^{f}\end{array}$ \\
\hline Conglomerado 1 - Semejanza promedio: 78,2 & Ch 21 & & & & \\
\hline Sistema de control de heladas (\% del área) & 100 & 88,75 & 23,06 & 29,48 & 29,48 \\
\hline Riego por goteo (\% de área con goteo) & 100 & 100 & 12,82 & 16,39 & 45,87 \\
\hline Mano de obra transitoria (jornales) & 500 & 480 & 10,94 & 13,98 & 59,85 \\
\hline Empaque (no: 0; en forma asociada: 1; propio: 2) & 1 & $1(72,7)$ & 9,60 & 12,28 & 72,13 \\
\hline Densidad de plantación de cerezos (árboles/ha) & 2940 & 1795 & 7,77 & 9,94 & 82,06 \\
\hline Conglomerado 2 - Semejanza promedio : 75,4 & LA 14 & & & & \\
\hline Empaque (no: 0; en forma asociada: 1; propio: 2) & 1 & $1(88,9)$ & 28,66 & 38,00 & 38,00 \\
\hline Asesoramiento (no: 0; ocasional: 1; permanente: 2) & 2 & $2(61,1)$ & 21,28 & 28,21 & 66,21 \\
\hline Área total con cerezos (ha) & 6,00 & 5,50 & 10,92 & 14,48 & 80,70 \\
\hline Conglomerado 3 - Semejanza promedio : 76,1 & LA 33 & & & & \\
\hline Riego por goteo (\% de área con goteo) & 100 & 100 & 29,68 & 38,98 & 38,98 \\
\hline Asesoramiento (no: 0; ocasional: 1; permanente: 2) & 2 & $2(53,3)$ & 18,76 & 24,64 & 63,62 \\
\hline Densidad de plantación de cerezos (árboles/ha) & 1250 & 2003 & 16,63 & 21,84 & 85,46 \\
\hline Conglomerado 4 - Semejanza promedio : 72,7 & S 15 & & & & \\
\hline Riego por goteo (\% de área con goteo) & 100 & 100 & 17,73 & 24,38 & 24,38 \\
\hline Empaque (no: 0; en forma asociada: 1; propio: 2) & 1 & $1(71,4)$ & 16,90 & 23,24 & 47,61 \\
\hline $\begin{array}{l}\text { Mano de obra permanente (cantidad de } \\
\text { empleados) }\end{array}$ & 2 & 2,29 & 9,90 & 13,61 & 61,22 \\
\hline Densidad de plantación de cerezos (árboles/ha) & 933 & 1803 & 9,51 & 13,08 & 74,31 \\
\hline Mano de obra transitoria (jornales) & 620 & 333 & 9,15 & 12,58 & 86,89 \\
\hline
\end{tabular}

S15 para las tipologías 3 y 4 , respectivamente). Ambas muestras prediales son muy similares a sus correspondientes FVG para las variables que contribuyeron al menos al $60 \%$ de la semejanza intragrupal. Pero ambas difieren de los respectivos promedios en densidad de plantación de cerezos. La contribución de esta variable a la semejanza grupal media fue de $21,84 \%$ y $13,08 \%$ para la finca LA33 y S15, respectivamente.

La selección de fincas representativas para los grupos 5 y 6 (LA7 y S6, respectivamente), fue facilitada por el pequeño número de muestras en estos grupos y la semejanza de los valores de las variables clave utilizadas para la clasificación. Por estas razones estos resultados no se incluyeron en la Tabla 4.

Para los agrupamientos más grandes (tipologías 1, 2 y 3) podría ser interesante elegir más de una finca piloto representativa para representar mejor la variabilidad observada dentro de cada conglomerado. El proceso de selección de las muestras prediales representativas se facilita con el uso del gráfico EMD. La FVG representa el "centro" del conglomerado y puede ser utilizada como referencia para guiar la selección de las muestras más representativas.

\section{CONCLUSIONES}

El presente trabajo logró diferenciar claramente tipos de fincas con características muy diferenciadas. Esto es de suma utilidad para seleccionar fincas piloto en programas de investigación y/o de extensión, ya que permite trabajar desde un principio con las más representativas de los conglomerados de mayor interés para las distintas instituciones (INTA, Universidades, gobiernos provinciales, etc.) y hacer un uso eficiente de los limitados recursos disponibles para intervenir. En este proceso de selección no se desconoce que existan otros tipos de fincas con necesidades diferentes, pero la metodología constituye una herramienta para la priorización.

También un estudio como el presentado en este artículo es una herramienta fundamental para diseñar estrategias de intervención específicas y coherentes para cada tipología predial, de modo de maximizar las posibilidades de tener impacto. A partir de los resultados de este estudio deberá 
profundizarse el análisis utilizando metodologías participativas, de modo de ahondar en los objetivos no explícitos que tienen los productores y evaluar alternativas productivas aceptables por ellos.

En futuras investigaciones debería analizarse la relación entre la implementación de medidas políticas de intervención (Ej., líneas de crédito, regulaciones comerciales y laborales, promociones, etc.), la toma de decisiones por parte de los productores y los efectos en la sustentabilidad de los sistemas. Estos estudios deberían basarse en modelos de simulación, pero para su formulación y evaluación es fundamental que se incluya a los actores más relevantes desde el inicio del proceso.

\section{AGRADECIMIENTOS}

Este trabajo ha sido realizado como parte del Proyecto EULACIAS (INCO-dev), Sexto Programa Marco de la Unión Europea, Contrato Nº 0032387.

\section{BIBLIOGRAFÍA}

Blazy, J.M.; M. Dorel, F. Salmon, H. Ozier-Lafontaine, J. Wery and P. Tixier, 2009a. Model-based assessment of technological innovation in banana cropping systems contextualized by farm types in Guadeloupe. European Journal of Agronomy 31:10-19.

Blazy, J.M.; H. Ozier-Lafontaine, T. Dorè, A. Thomas and J. Wery, 2009b. A methodological framework that accounts for farm diversity in the prototyping of crop management systems. Application to banana-based systems in Guadeloupe. Agricultural Systems 101:3041.

Bray, J.R. and J.T. Curtis, 1957. An ordination of the upland forest communities of Southern Wisconsin. Ecol. Monogr. 27:325-349.

Catalán, I.A.; M.T. Jiménez, J.I. Alconchel, L. Prieto and J.L. Muñoz, 2006. Spatial and temporal changes of coastal demersal assemblages in the Gulf of Cadiz (SW Spain) in relation to environmental conditions. Deep Sea Research Part II: Topical Studies in Oceanography 53:1402-1419.

Cittadini, E.D, 2005. FRUPAT: Generador de Coeficientes Técnicos (GCT) para sistemas frutícolas de Patagonia Sur. CD-rom, ISBN: 987-521-160-5. EEA Chubut (INTA), Argentina - PPS (WUR), Holanda.

Cittadini, E.D.; H. van Keulen and P.L Peri, 2006. FRUPAT: a tool to quantify inputs and outputs of Patagonia fruit production systems. Acta Horticulturae 707:223-230.

Cittadini, E.D.; C.E. Sanz, S.E. Szlápelis, F.A. Manavella, L. San Martino, M. Muñoz, A.B. Pugh, M.Á. Cárcamo, C.A. Mundet, P.L. Peri, N. de Ridder, N. Kikuchi, J.A. Nancucheo and $\mathrm{H}$. van Keulen, 2008. Sweet Cherry Production in South Patagonia, Argentina. Acta Horticulturae 795: 585-590.
Clarke, K.R., 1993. Non-parametric multivariate analyses of changes in community structure. Aus. J. Ecol. 18:117-143

Clarke, K.R. and R.M. Warwick, 2001. Change in marine communities: an approach to statistical analysis and interpretation, $2^{\text {nd }}$ edition. PRIMER-E: Plymouth, 170 pp.

Everitt, E., 1980. Cluster Analysis, 2nd ed. Heinhemann, London.

Field, J.C.; K.R. Clarke and R.M. Warwick, 1982. A practical strategy for analysing multispecies distribution patterns. Mar. Ecol. Prog. Ser. 8:37-52.

Gaspar, P.; M. Escribano, F.J. Mesías, A. Rodriguez de Ledesma and F. Pulido, 2008. Sheep farms in the Spanish rangelands (dehesas): Typologies according to livestock management and economic indicators. Small Ruminant Research 74:52-63.

Iraizoz, B.; M. Gorton and S. Davidova, 2007. Segmenting farms for analysing agricultural trajectories: A case study of the Navarra region in Spain. Agricultural Systems 93:143-169.

Joffre, O.M. and R.H. Bosma, 2009. Typology of shrimp farming in Bac Lieu Province, Mekong Delta, using multivariate statistics. Agriculture, Ecosystems \& Environment 132:153-159.

Kobrich, C.; T. Rehman and M. Khan, 2003. Typification of farming systems for constructing representative farm models: two illustrations of the application of multivariate analyses in Chile and Pakistan. Agricultural Systems 76:141-157

Kostrowicki, J., 1977. Agricultural typology concept and method. Agricultural Systems 2: 33-45.

Kruskal, J.B, 1964. Multidimensional scaling by optimizing goodness of fit to a non-metric hypothesis. Psychometrika 29:1-27.

Kruskal, J.B. and M. Wish, 1978. Multidimensional scaling. Sage Publications, Beverly Hills, California, 96 pp.

Maseda, F.; F. Díaz and C. Álvarez, 2004. Family Dairy Farms in Galicia (N.W. Spain): Classification by Some Family and Farm Factors Relevant to Quality of Life. Biosystems Engineering 87:509-521

Milán, J.M.; J. Bartolomé, R. Quintanilla, M.D. GarcíaCachán, M. Espejo, P.L. Herráiz, J.M. Sánchez-Recio and J. Piedrafita, 2006. Structural characterisation and typology of beef cattle farms of Spanish wooded rangelands (dehesas). Livestock Science 99:197-209.

Muñoz, M., 2004. Relevamiento de lotes productivos en la localidad de Los Antiguos. Informe Técnico, Consejo Agrario Provincial - Delegación Los Antiguos, Argentina, $7 \mathrm{pp}$.

Nahed, J.; J.M. Castel, Y. Mena and F. Caravaca, 2006. Appraisal of the sustainability of dairy goat systems in Southern Spain according to their degree of intensification. Livestock Science 101:10-23.

Olaizola, A.M.; T. Chertouh and E. Manrique, 2008. 
Adoption of a new feeding technology in Mediterranean sheep farming systems: Implications and economic evaluation. Small Ruminant Research 79:137-145.

Pacini, G.C.; E. Righi, J. van der Burgh, K. Bitchibaly, E. Yeboah, D. Nwaga, K.C. Kaizzi, A.K. Katwijukye, D. Mugendi, A. Mwijage, P. Mapfumo and M. Mwale, 2006. Deliverable of the project Africa NUANCES. D.6.2.1. Generic typologies for categorizing and describing household diversity and livelihood strategies, $51 \mathrm{pp}$.

Poussin, J.C.; A. Imache, R. Beji, P. Le Grusse and A. Benmihoub, 2008. Exploring regional irrigation water demand using typologies of farms and production units: An example from Tunisia. Agricultural Water Management 95:973-983.

Righi, E.; G.C. Pacini, E.D. Cittadini, M. Perales, S. Dogliotti, V. Cuevas, V. Aguerre and C. Vazzana, 2009. Deliverable of the project EULACIAS. D5.2.1. Farm typology for each case study region.
Righi, E.; S. Dogliotti, F.M. Stefanini and G.C. Pacini, 2010. Capturing farm diversity at regional level to upscale farm level impact assessment of sustainable development options. Agric. Ecosyst. Environ. doi:10.1016/j.agee.2010.07.011

Sanz, C., 2005. Relevamiento del sector cerecero del Valle Inferior del Río Chubut. Informe Técnico, INTA EEA Chubut, Argentina, 8 pp.

Selleslagh, J. and R. Amara, 2008. Environmental factors structuring fish composition and assemblages in a small macrotidal estuary (eastern English Channel). Estuarine, Coastal and Shelf Science 79:507-517.

Stergiou, K.I.; D.K. Moutopoulos; M.C. Soriguer; E. Puente; P.G. Lino; C. Zabala; P. Monteiro; L.A. Errazkin and K. Erzini, 2006. Trammel net catch species composition, catch rates and métiers in southern European waters: A multivariate approach. Fisheries Research 79:170182. 